\title{
Evaluation of Gamma-Ray Attenuation Parameters for Some Materials
}

\author{
Amal A. El- Sawy \\ Radiation Safety Department, Egyptian Nuclear and Radiological Regulatory \\ Authority (ENRRA)3 Ahmed El-Zomor St., Nasr City, 11762, P.O. Box 7551, \\ Cairo- Egypt
}

\begin{abstract}
In the present study, the linear attenuation coefficient and mass attenuation coefficient have been calculated at energy range of $0.8 \mathrm{MeV}, 1 \mathrm{MeV}, 3 \mathrm{Mev}, 5 \mathrm{MeV}$ and $7 \mathrm{MeV}$. The mass attenuation Coefficient $\mu_{m}$ has been calculated analytically for Iron material $\mathrm{Fe}(\rho=$ $\left.7.87 \mathrm{~g} / \mathrm{cm}^{3}\right)$ and developed material lead $P b\left(\rho=11.3 \mathrm{~g} / \mathrm{cm}^{3}\right)$ at photon energies $[0.8 \mathrm{MeV}$ to $7 \mathrm{MeV}]$ using $X$-Com computer code. The obtained results were compared with the calculated values. The linear attenuation Coefficient $\mu$ has been Calculated, its variations with photon energy were plotting. The Comparison between two materials Iron and Lead were also studied. The obtained result shows that $\mu$ and $\mu \mathrm{m}$ depends on the photon energy, density of the materials and atomic number. From this studs we notice that the values of $\mu_{m}$ and $\mu$ of Lead material is greater than Iron Material and also the values are decreasing with increasing the photon emergy. The half value layer $(H V L)$ and tenth value layer (TVL) of two studied materials were calculated at photon energy range of $0.8 \mathrm{MeV}$, $1 \mathrm{MeV}, 5 \mathrm{MeV}$ and $7 \mathrm{MeV}$. The relation between this equation $\mathrm{Ln}\left(\mathrm{I}_{\mathrm{o}} / \mathrm{I}\right)$ and the thickness of material were calculated, so the curves Show that the relation of $L_{n}\left(I_{d} / I\right)$ of Iron and Lead materials is increasing with the increasing of material thickness and the relation gives the straight line atso the comparison of this relation for Lead material is higher than Iron material. Shis stud can also be utilized to improve the effectiveness of radiation shielding by using the developed shielding material.

Keywords: Linear attenuation coefficient $(\mu)$, Mass attenuation coefficient $\left(\mu_{m}\right)$, Half layer value (HVL), Tenth layer value (TVL), X-Com computer code, Gamma-ray.
\end{abstract}

\section{Introduction}

Gamna rays are electromagnetic waves. They are produced by many radioactive substances. They can also be found in nuclear reactors and in cosmic radiation. There are many useful applications of gamma ray such as radiotherapy, medical tracer and sterifization. On the other hand, when $\gamma$ - rays are absorbed by a living organism (e.g. human), they may causes serious effects. Therefore, it is necessary to find out some substances that can effectively absorb and block $\gamma$ - rays [1]. In order to ensure radiation safety in the various application of ionizing radiation technology certain procedures must be put in place to reserve exposure level to their maximum. These procedures includes, designing work schedule in way that the safest possible distance in kept from the source. However, there are limitations to the above procedures [2].

Perhaps the most effective radiation protection is the use of shielding materials between the workers and the source and also to curtail the radiation to where it is being applied 
without constituting danger to the general public. A shield material is expected to have high gamma ray attenuation coefficient in orders that a small thickness will produce significant reduction in intensity.

Due to the development of nuclear technology with time, various beneficial applications of different types of radiations in medicine, industry, agriculture and research as well as for nuclear power generation are increasing day by day. But a drawback to these peaceful uses of radiation is that if it is exposed to the personnel including other human beings, who are in vicinity of it beyond its permissible dose limit, a deleterious effect may be observed in them $[3,4]$. Therefore, the radiation must be attenuated enough to protect the personnel from the harmful effects caused by it and also enable them to work by using an opposite shielding material. The shielding used for this purpose is generally known as biological shielding [5, 6].

To design and choose an appropriate biological shielding it is necessary to have known its nuclear, structured and physical properties and also the characteristics of radiation impinging on it. The nuclear parameters that must be known to design and choose a shielding material are means attenuation coefficient $\left(\mu_{\mathrm{m}}\right)$, linearr attenuation coefficient $(\mu)$ for gamma rays which is related to half value layer (HVL), Tenth value lave (TVL) $[7,8]$. It is important to determine the values of different shielding parameters in various ways to know the shielding effectiveness of the shielding materials developed with time $[9,10$, and 11].

The probability of photon interacting in a particular way with a given material, per unit path length, is called the linear atteruation coefficient [12]. The linear attenuation coefficient per unit mass of the materiat is expressed as mass attenuation coefficient to avoid the effects of variations in the density of a naterial for reference purposes [13].The main objectives of the present study âre to determine the values of above parameters and to represent a comparison of shielding effectiveness of the different materials used in the present work at different photon energy:

In this work, Mont Calo program MCNP5 was written to simulate the transport of gamma-rays through the matter. The gamma-ray intensity (I) of Iron shield material were calculated and the developed material lead according different thickness and energy range were calculated theoretically using MCNP model. The Comparison between two materials Iron and Lead were llso studied.

\section{Theoretical Background}

\subsection{Attenuation of Radiation in Matter}

Wher radiation interacts with matter, its intensity will decreases as it travel through a material The attenuation properties of radiation will affect how much shielding is necessary and how much dose receives.

\subsection{Calculation of Mass Attenuation Coefficient}

When gamma rays of intensity (I) is passing through a material thickness (dx) the resulting reduction $(\mathrm{dI})$ in the intensity is termed attenuation. This decreasing (dI) is proportional to both (I) and (dx), and is expressed as:

dIa Idx

$$
\mathbf{d I}=-\mu I d x
$$

(1) 
Where $\mu$ is the linear attenuation coefficient, which depends on the absorbing material and the energy of gamma rays.

By integration the equation (2) resulting [14] to:

$$
I=I_{0} e_{-}{ }^{\mu x}
$$

Equation (3) is called linear attenuation formula; this equation is referred to as Lambert's low where:

$\mathrm{I}=$ the shielded dose rate.

$\mathrm{I}_{0}=$ the initial dose rate.

$\mu=$ the linear attenuation coefficient in $\left(\mathrm{cm}^{-1}\right)$.

Since the beam intensity attenuation is due to the interaction of photons with electrons, of atoms of the object, the attenuation coefficient is therefore dependent upon atomic number of the atoms and energy of the photons. There are two main features of the limear attenuation coefficient, first the linear attenuation coefficient increase as the atomic number of the absorber increases, second the linear attehuation coefficient for all materials decreases with the energy of the gamma rays Finally, the linear attenuation coefficient is a function of the atomic number $z$, andenergy $E$ [15].

Linear attenuation coefficient $\mu$ is always proportional to the density of the materials. This is:

Where $\mu_{\mathrm{m}}$ is called mass attenuation coefficient.

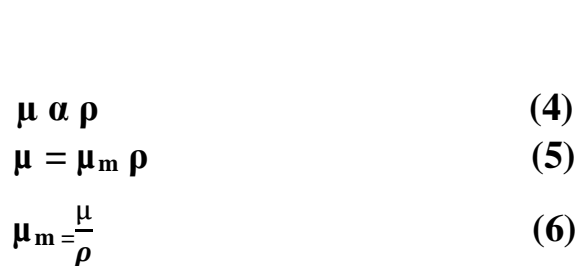

The mass attenuation goefficient is defined as the ratio of linear attenuation and absorber density $(\mu / \rho)$ When radiation passes through matter, it dissipates its energy in the ionization and excitation of the molecules of the materials, either directly as we have for charged particles or indirectly as we have for neutrons and photons. Radiation protection methods are based on measurable physical and chemical changes resulting from these ionization ard excitations [16].

\subsection{Calculation of HVL and TVL}

Half value layer (HVL) is the thickness of a shield or an absorber that reduces the radiation lever by a factor 2 that is to half the initial level and is calculated by the following equation:

$$
\text { HVL }=\frac{\ln 2}{\mu}=\frac{0.693}{\mu}
$$

Where $\mu\left(\mathrm{cm}^{-1}\right)$ is the linear attenuation coefficient of the absorber. There are two main features of the half value layers. First, the half value layer decreases as the atomic number (z) of the absorber decreases. Second, the half value layer for all materials increases with increasing the gamma rays energy [17].

Similarly, Tenth value layer (TVL) is defined as the thickness of a shield required for attenuating a radiation beam to $10 \%$ of its radiation level and is computed by,

$$
\text { TVL }=\frac{\ln 10}{\mu}=\frac{2.3026}{\mu}
$$




$$
\begin{aligned}
& \mu=\frac{1}{x} \ln \frac{I_{0}}{I} \\
& \ln \frac{I_{0}}{I}=\mu \mathrm{x}
\end{aligned}
$$

The $\mu, \mu_{\mathrm{m}}$, HVL and TVL values have also calculated at the different photon energy for shielding materials. The equation of $\ln \frac{I_{0}}{I}$ as a function of shielding thickness at different energy level were also calculated.

\section{Method of Calculation}

\subsection{Mathematical Model}

MCNP is a general purpose radiation transport code for modelling the interaction of radiation with materials and also tracks all particles at all energies. In this study, MCNP5 simulation model was written to simulate the transport of gamma-rays through the absorber material. A point isotropic source emitted photons surrounded by an Iron annular spherical shell $30 \mathrm{~cm}$ in thickness. The point detector was placed at a considering point $160 \mathrm{~cm}$ away from the source to calculate the photon intensity (I). The Comparison between two materials Iron and Lead at differen thickness $(5 \mathrm{~cm}$ to $30 \mathrm{~cm})$ and energy range $(0.8 \mathrm{MeV}$ to $7 \mathrm{MeV})$ were also studied.

\subsection{Calculation of Linear Attenuation Coefficient and Mass Attenuation Coefficient}

The linear attenuation coefficient $\left(\mathrm{cm}^{-1}\right)$ for two Shielding materials Iron, and Lead have been calculated analytically using the eqn. (3) at photon energy range from $0.8 \mathrm{MeV}$ to $7 \mathrm{MeV}$. The mass attenuation coefficient $\left(\mu_{\mathrm{m}}\right)$ was compared with the calculation obtained using X-Com computen code. The X-Com is a data base and its run on a $\mathrm{X}$-Com code composites contents are input and output is the mass attenuation coefficient $(\mu / \rho)$. The photon attenuation have been evaluated comparing $I$ and $I_{0}$ which are the measured count rates in detector, respectively, with and without the absorber of thickness $\mathrm{x}(\mathrm{cm})$.

\section{Results and Discussion \\ 4.1 Linear Attenuation Coefficient $(\mu)$}

The linear attenuation coefficient $\mu\left(\mathrm{cm}^{-1}\right)$ as a function of photon energy for two types of materials are presented in fig. 1. From this figure, it is clear that the linear attenuation coefficint is decreasing rapidly at low energy but in high energy it decreases slowly. The energy gependence of photon interaction with the material is also seen from this figure. It is due to different photon absorption from different energy range. The photon absorption process is mainly at low energy than in high energy. From this curve it is clear that the linear attenuation coefficient for Lead material is higher than Iron material and there is a large difference in low energy than in high energy. This is due to the high density and atomic number of Lead. It means that the linear attenuation coefficient depends on the photon energy, density and atomic number of material. 


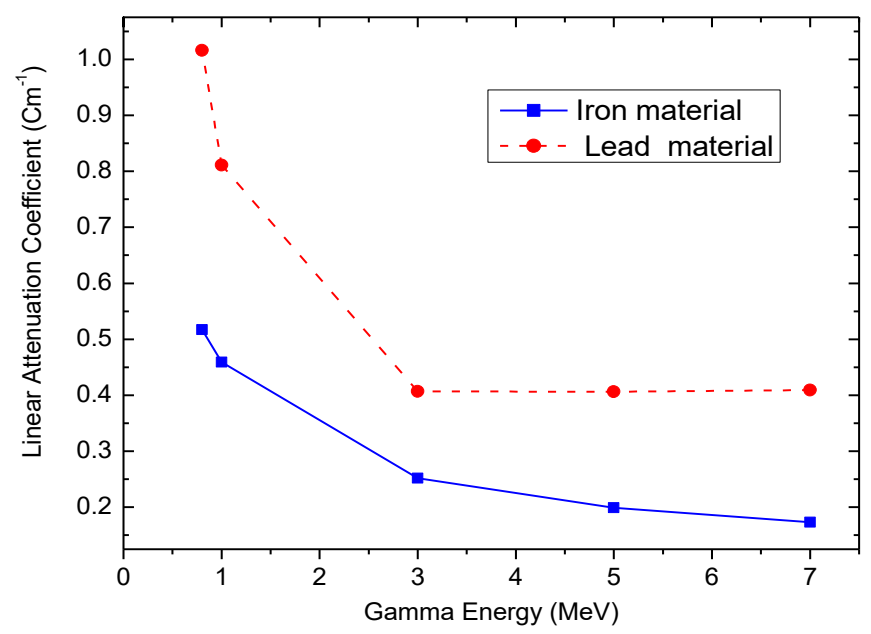

Figure 1. Comparison of Linear Attenuation Coefficient of Gamma Radiation versus Energy for Iron and Lead Material

\subsection{Mass Attenuation Coefficient $\left(\mu_{\mathrm{m}}\right)$}

The theoretically calculated values of mass attenuation coefficient $\mu_{\mathrm{m}}\left(\mathrm{cm}^{2} / \mathrm{g}\right)$ using the eqn. (6) and the X-Com values of Iron and Lead materials for gamma rays of energy range from $0.8 \mathrm{MeV}$ to $7 \mathrm{MeV}$ were shown in table (1) From this table, it is seen that the calculated and X-Com values of $\mu_{\mathrm{m}}$ are in good greement, we notice that the values are very close at low energy than high energy due to different photon absorption from different energy range.

Table 1. The theoretical and X-Côm Values of Mass Attenuation Coefficient

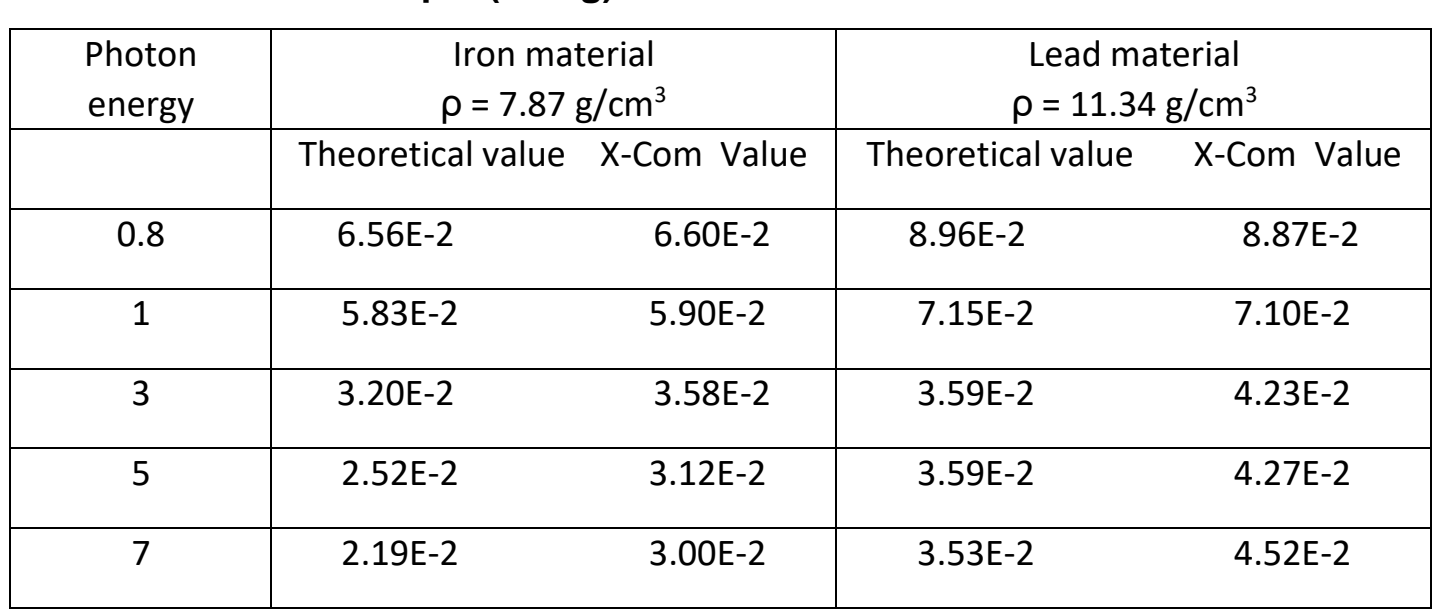


Figure 2 represents the variation of mass attenuation coefficient $\mu_{\mathrm{m}}$ with photon energy of two types of materials Iron $(\mathrm{Fe})$ and Lead $(\mathrm{Pb})$. From this figure, it is clear that the mass attenuation coefficient depends on the incident photon energy and composition of the materials and its decreases with increasing the photon energy. This figure show that the mass attenuation coefficient of Lead material is higher than Iron material, this is due to the density of Lead $\left(11.34 \mathrm{~g} / \mathrm{cm}^{3}\right)$ is higher than the density of $\operatorname{Iron}\left(7.87 \mathrm{~g} / \mathrm{cm}^{3}\right)$ and the Lead has an atomic number (81) higher than Iron (26). It is conclude that the mass attenuation coefficient depends on the density and the atomic number of material.

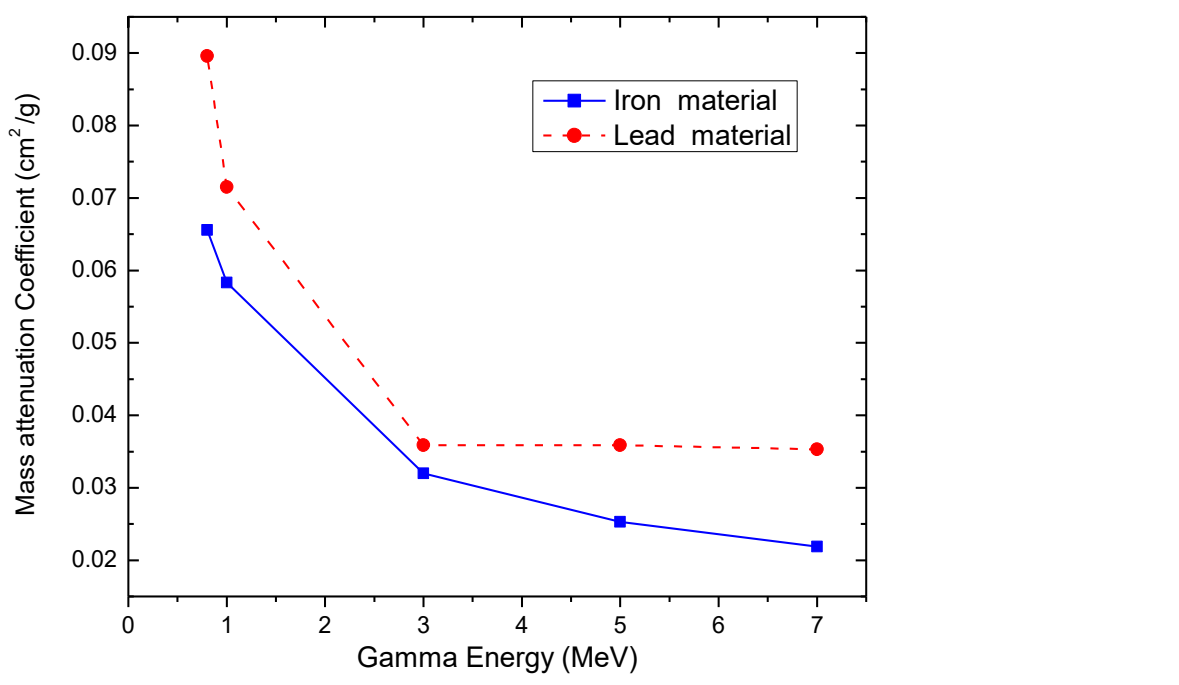

Figure 2. Comparison of Mass Attenuation Coefficient of Gamma Radiation versus Gamma Energy for Iron and Lead Material

Table 2 displayed the calculated mass attenuation coefficient $\mu_{\mathrm{m}}$ and linear attenuation coefficient $\mu$ for two materials Iron and Lead at different energy range from $0.8 \mathrm{MeV}$ to 7 $\mathrm{MeV}$. From this table we notiee that the mass attenuation coefficient and linear attenuation coefficient weecreasing with increasing the energy, and these values for Lead material are highe than in Iron material due to the high density of Lead than Iron.

Table 2. Linear Attenuation Coefficient $\mu\left(\mathrm{cm}^{-1}\right)$ and Mass Attenuation coefficient $\mu \mathrm{m}\left(\mathrm{cm}^{2} / \mathrm{g}\right)$ of Iron and Lead Material at Energy Rang (0.8 MeV to $7 \mathrm{MeV}$ )

\begin{tabular}{|c|c|c|c|c|}
\hline $\begin{array}{l}\text { Photon energy } \\
\text { (MeV) }\end{array}$ & \multicolumn{2}{|c|}{$\begin{array}{c}\text { Iron material } \\
\rho=7.87 \mathrm{~g} / \mathrm{cm}^{3}\end{array}$} & \multicolumn{2}{|c|}{$\begin{array}{c}\text { Lead material } \\
\rho=11.34 \mathrm{~g} / \mathrm{cm}^{3}\end{array}$} \\
\hline & $\begin{array}{c}\mu\left(\mathrm{cm}^{-1}\right) \\
\left(\mathrm{cm}^{2} / \mathrm{g}\right)\end{array}$ & $\mu_{\mathrm{m}}$ & $\begin{array}{l}\mu\left(\mathrm{cm}^{-1}\right) \\
\left(\mathrm{cm}^{2} / \mathrm{g}\right)\end{array}$ & $\mu_{m}$ \\
\hline 0.8 & 0.517 & 0.0656 & 1.016 & 0.0896 \\
\hline 1 & 0.459 & 0.0583 & 0.811 & 0.0715 \\
\hline 3 & 0.252 & 0.0320 & 0.407 & 0.0359 \\
\hline 5 & 0.199 & 0.0252 & 0.407 & 0.0359 \\
\hline 7 & 0.173 & 0.0219 & 0.409 & 0.0353 \\
\hline
\end{tabular}




\subsection{Half Value Layer (HVL), Tenth Value Layer (TVL) and Ln ( $\left.I_{0} / I\right)$}

Half value layer (HVL) and Tenth value layer (TVL) are two important parameters in designing any radiation shielding since half value layer and tenth value layer indicates the required thickness of an absorber to reduce the radiation level to half and one tenth. The calculated HVL and TVL of Iron and Lead materials at energy range $0.8 \mathrm{MeV}, 1 \mathrm{MeV}$, $5 \mathrm{MeV}$ and $7 \mathrm{MeV}$ have been shown in table 3. It can be seen that the HVL and TVL are increasing by increasing the energy and the Lead material has a lower HVL and TVL than Iron material. It means that at the same energy of incident radiation, a thickness of lead will be required to attenuation gamma radiation; lead will be required to attenuation to half its original intensity and to tenth its original intensity. This means that we needed a small thickness to attenuate the intensity for lead material, so it concludes that the Lead material is the best for shielding of gamma- ray.

Table 3. Half Value Layer (HVL) and Tenth Value Layer (TVL) of Iron and Lead Material Energy Rang (0.8 MeV to $7 \mathrm{MeV}$ )

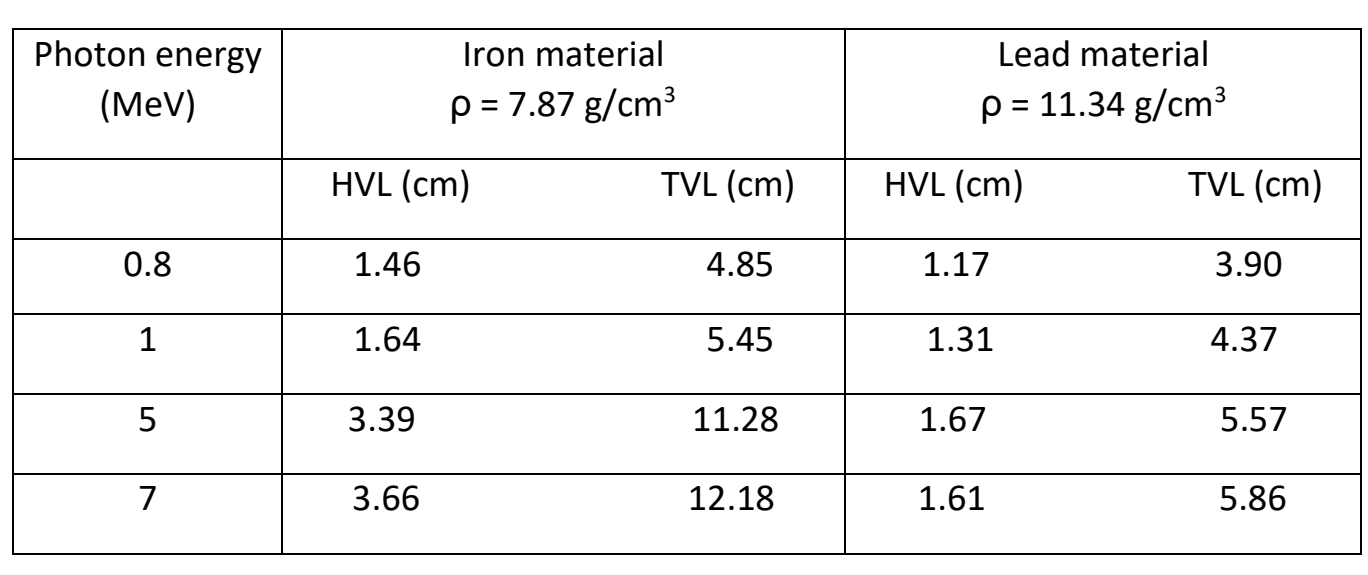

Figure 3 and 4 are epresenting the relation between $\operatorname{Ln}\left(\mathrm{I}_{0} / \mathrm{I}\right)$ and different thickness $(\mathrm{cm})$ at energy $0.8 \mathrm{MeV}$ and $7 \mathrm{MeV}$ for Iron and Lead materials. From these figures we notice that the relation gives straight line. In fig. 3 we also seen that at energy $7 \mathrm{MeV}$, the Ln $\left(\mathrm{I}_{0} / \mathrm{I}\right)$ is increasing wi $\mathrm{b} /$ the material thickness increasing and the values at small thickness for two mater als are close then it is increasing at large thickness. Also we saw that the $\operatorname{Ln}\left(I_{0} / I\right)$ for 1 ead material is higher than in Iron material. While fig. 4 represents the reation at $0.8 \mathrm{MeV}$. From this figure, we seen that the relation gives straight line, it is increasing with increasing the thickness of materials and the increasing of the plotgives the same values at small and large thickness of Iron and Lead materials. 


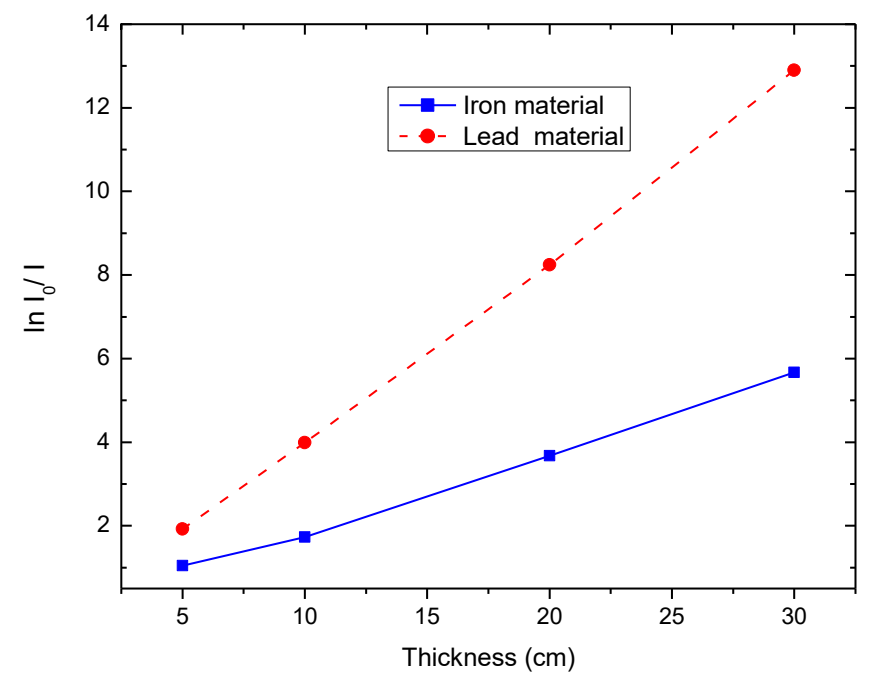

Figure 3. A plot Im I / Against Thickness for Iron and Lead Material at Energy $7 \mathrm{MeV}$

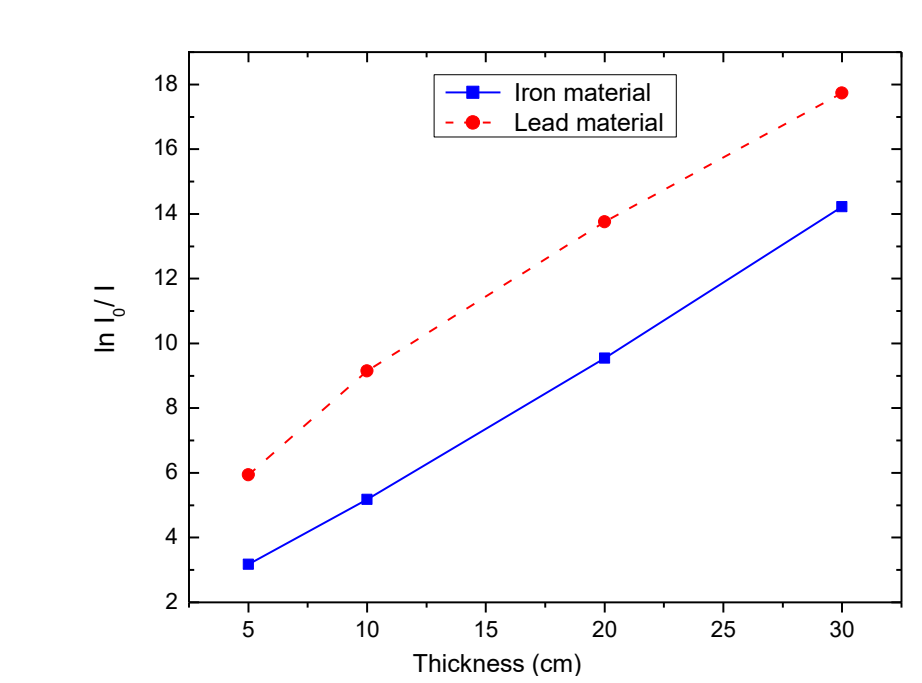

Figure 4. A plot lo $_{0}$ / Against Thickness for Iron and Lead Material at Energy $0.8 \mathrm{MeV}$

The plotting of variation of $\mathrm{Ln}\left(\mathrm{I}_{\mathrm{o}} / \mathrm{I}\right)$ with material thickness for Iron and Lead at energy range of $0.8 \mathrm{MeV}, 1 \mathrm{MeV}, 5 \mathrm{MeV}$ and $7 \mathrm{MeV}$ were shown in fig. 5 and 6 . From these figures, it is clear that the relation gives the straight line. Also we have seen that the value of $\operatorname{Ln}\left(\mathrm{I}_{0} / \mathrm{I}\right)$ is high at low energy, while it's decreasing with the increasing of photon energy. Also the relation value in all energy range from $0.8 \mathrm{MeV}$ to $7 \mathrm{MeV}$ is low at small thickness then it is increasing with the increasing of the material thickness. 


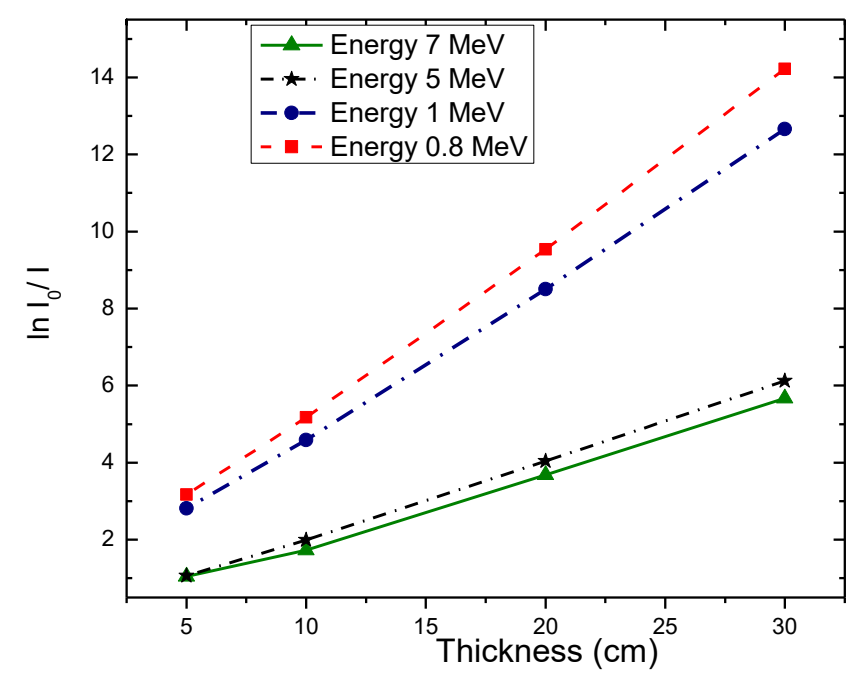

Figure 5. A plot In Io /I Against Thickness for Iron Material for Different Energy Range

\section{Conclusion}

Figure 0. A plot In I0 / Against Thickness for Iron Material for Different Energy Range

This study concludes that the shielding effectiveness of any shielding material depends on its density and the atomic number. To design and select an appropriate shielding material, all the nuclear parameters associated with it should be studied carefully. In the current research, the gamma-ray attenuation parameters were studied for Iron and Lead material. In this study the linear attenuation coefficient and mass attenuation coefficient of gamma-ray for Iron and Lead material were calculated at $0.8 \mathrm{MeV}$ to $7 \mathrm{MeV}$, Attenuation coefficient decreases with increasing energy and density of material. It was observed that terms of radiation shielding the lead material was more suitable than Iron material because it has a high atomic number $(\mathrm{z}=82)$ and heavy density $(\rho=11.3)$. The half value layer and tenth value layer were calculated, it can be seen that the Lead has a lower HVL and TVL than Iron. It concluded that lead has a higher attenuation coefficient, higher 
density, lower half value layer and lower tenth value layer than Iron; this means that it has a good absorber of radiation.

This study can also be utilized for improving the shielding performance of Iron by using the Lead material. This theoretical Study can be taken as a biological shield in the future. In addition to that, it can be recommended to study the changes of attenuation properties of both Iron and Lead material with the changes of atomic number present in it, and to develop a simplified experimental model for neutron and gamma-ray transport calculations.

From this work, It is concluded that while the Iron is an ideal shielding material, Lead is better than Iron for using in shielding materials.

\section{References}

[1] Auwal M. M et al, "Determination of Absorbed and Effective Dose From Natural Background Radiation" around a Nuclear Research Facility", International Archive of Applied Science and Technology Vol.2 [1], 23-27, 2011.

[2] Ero F. A and Adebo B. A, "Determination of $\gamma$ - Radiation characteristics of Some Woods in Western Nigeria", International Archive of Applied Science and Technology, Vol.3 [2], 14-20, June.2012.

[3] Ripan Biswas et al, "Calculation of Gamma- ray Attenuation Parameter for Locally Developed Shielding Material: Polyboron", Journal of Radiation Research and Applied Science 9, 26-34, 2016.

[4] Akkurt, I., Akyıldırım, H., Mavi, B., Kilincarslan, S., \& Basyigit, C. "Photon attenuation coefficients of concrete includes barite in different rate", Annals of Nuclear Energy, 37,910e914, 2010.

[5] El-Khayatt, A. M., "Radiation shielding of concretes containing differen lime/silica ratios", Annals of Nuclear Energy, 37, 991e995, 2010.

[6] El-Khayatt, A. M., \& Akkurt, I., "Photon interaction, energy absorption and neutron removal cross section of concrete including marble", Annals of Nuclear Energy, 60, 8e14, 2013.

[7] Elmahroug, Y., Tellili, B., \& Souga, C., "Calculation of gamna and neutron shielding parameters for some materials polyethylene-based", International Journal of Physics and Research, 3, 33e40, 2013.

[8] Hubbell, J. H., \& Seltzer, S. M., "Tables of X-ray Mas attenuation coefficients and mass energyabsorption coefficients $1 \mathrm{keV}$ to $20 \mathrm{MeV}$ for elements $Z 1 / 4$ to 92 and 48 additional substances of dosimetric interest", NISTIR-5632. Gaithersbutrg: Natonal Institute of Standards and Technology, 1995.

[9] Kucuk, N., Cakir, M., \& Isitman, N. A., "Mass attenuation coefficients, effective atomic numbers and effective electron densities for some polymers", Radiation Protection Dosimetry, 1e8, 2012.

[10] Madhusudhan Rao, A. S. Narender, K., Gopal Kishan Rao, K., Krishna, G. N., \& Murthy, R. K., "Mass attenuation coeffieients, effective atomic and electron numbers of alkali halides for multi-energetic photons", Research Journal of Physical Sciences, 1(6), 11e16, 2013.

[11] Iqbal, S. M., Ranman, A., Fakarudin, M., Nor Paiza, M. H., \& Ismail, M., "Mass attenuation coefficients, effective atomic and electron-numbers of stainless steel and carbon steels with different energies", Jurnal Sains Nuklear Malaysia, 23(2), 19e25, 2011.

[12] I. I. Bashter, A. el-Sa ed Abdo, M. Samir Abdel-Azim, "Magnetite Ores with Steel or Basalt for Concrete Radiation Snielding", Jpn. J. Appl. Phys, Vol. 36, Part1, No. 6A, 1997.

[13] I. Akkurt, K. Gunoglu, C. Basyigit, S. Lilincarslan and A. Akkas, "Cement Paste as a Radiation Shielding Material", ACTA Physics Polonica A, Vol. 123, 2013.

[14] K. Sakr, et al, Effect of High Temperature or Fire in Heavy Weight Concrete Properties", Cement and Concrete Research, Vol. 35, PP.590-596, 2005.

[15] 15- Elias, S et al, "Characterization of Rubber Using $\gamma$ - Ray Attenuation Technique", In Proceeding, UKM Bangi, Malaysia, 196-206,1990.

[16] 16-Shielding of Gamma Radiation - Nuclear Power,http://www.nuclear-power.net/nuclearpower/reactor-physics/atomic-nuclear power, 2013

[1] ] 7- http://wwwen.wikibooks.org/wiki/basic_physics_of_Nuclear_Medicine Attenuation of Gamma Ray, 2015. 